\title{
IN VITRO SCOLICIDAL EFFECT OF BEE VENOM ON ECHINOCOCCUS GRANULOSUS PROTOSCOLICES
}

\author{
By
}

\author{
RANIA AYMAN TAWFIK \\ Department of Parasitology, Faculty of Medicine, Ain Shams University, Cairo \\ 11566, Egypt (e-mail: rania-tawfik@med.asu.edu.eg or ranya t@yahoo.com)
}

\section{Abstract}

Hydatid disease has significant economic and health impacts in many parts of the world including Egypt and the Middle East. Surgery remains the most efficient treatment, however, dissemination of protoscolex-rich fluid during surgery is a major cause of recurrence. Instillation of a scolicidal agent into hydatid cysts is the most commonly employed measure to prevent this complication. To date, various scolicidal agents have been tried with varying success.Many of these scolicidal agents may cause unacceptable side effects. The present study investigated the scolicidal effect of bee venom (BV) on Echinococcus granulosus protoscolices. Protoscolices were incubated with 50ppm and 100ppm BV for 30 minutes and scolicidal effect was assessed by motility, eosin exclusion test, morphological and ultrastructural alterations by light microscopy and Transmission Electron Microscopy (TEM). Apoptosis was determined by caspase-3 immunohistochemistry. Protoscolices incubated with 50ppm BV showed decreased viability by eosin exclusion test while those incubated with $100 \mathrm{ppm}$ BV showed a $100 \%$ death rate. BV caused distinct alterations in the morphology of protoscolices by both light microscopy and TEM and the expression of caspase- 3 in protoscolices incubated with BV was significantly high. The results of the present study suggested that BV can be a very promising scolicidal agent and further studies on its effects and safety in experimental animals are recommended.

Key words: In-vitro study, Echinococcus granulosus, Protoscolices, Bee venom.

\section{Introduction}

Cystic echinococcosis (CE) is a zoonotic parasitic disease caused by the larval stage of Echinococcus granulosus(E. granulosus). It is endemic and has significant economic and health impacts in many parts of the world including Egypt and the Middle East (Sadjjadi, 2006; El-Shazly et al, 2007). Dogs and other canids are the definitive hosts that harbour the adult tapeworms while herbivores act as intermediate hosts and become infected through ingestion of the parasite's eggs excreted in the feces of the definitive host. Humans acquire the infection by accidental ingestion of $E$. granulosus eggs (Sarkari and Rezaei, 2015). Hydatid cysts are mostly localized in the liver (50-80\%) while the second most common site is the lungs (5-30\%). Cysts have also been detected in the spleen, kidney, heart, bones, the central nervous system, and other organs but with less frequency (Sayek et al, 1980). Protoscolices inside the cysts are microscop- ic larvae capable of development to adult worms in the final host intestine or to secondary hydatid cysts in the intermediate host viscera (Sharafi et al, 2017).

There were four treatment options for hydatid disease: surgery, percutaneous aspiration, medical treatment and watch and wait (Bru-netti et al, 2010). Surgery remains the most efficient and widely used treatment modality, however, dissemination of protoscolex-rich fluid during surgery is a major cause of recurrence. Operative spillage may also lead to secondary disseminated intraperitoneal hydatidosis (Rajabi, 2009). Instillation of a scolicidal agent into a hepatic hydatid cyst is the most commonly employed measure to prevent this serious complication (Tozar et al, 2005). Till now, various scolicidal agents including plant extracts, Albendazole, hypertonic saline, hypertonic glucose, ethyl alcohol, silver and selenium nanoparticles were tried with various success (Pérez-Serrano et al, 1994; Besim et al, 
1998; Kayaalp et al, 2001; Hosseini et al, 2006; Moazeni and Nazer, 2010; Moazeni and Roozitalab, 2012; Mahmoudvand et al, 2014; Rahimi et al, 2015). Unfortunately, many of these scolicidal agents might cause unacceptable side effects, limiting their use (Moazeni and Alipour-Chaharmahali, 2011). Thus, finding new scolicidal agents with fewer side effects, low cost, and high efficacy was an urgent need for surgeons (Adas $e t$ al, 2009).

Honey bee venom (BV) is a liquid blend of biologically active substances comprising proteins, peptides, enzymes and other small molecules (Pak, 2017). These biologically active compounds contain various peptides such as melittin, apamin, adolapin and mast cell degranulating (MCD) peptide; enzymes such as phospholipase A2 (PLA2) and hyaluronidase as well as different biological amines including histamine and epinephrine (Moon et al, 2008; Zhou et al, 2010). Melittin is a small protein containing 26 amino acid residues and is the main bioactive component in BV. It has been reported to exert hemolytic and antimicrobial effects through membrane perturbations, constriction or dilatation of blood vessels, damage to enzyme systems and pro-inflammatory effects (Lee et al, 2007).

$\mathrm{BV}$ has long been used for treatment of rheumatoid arthritis and skin diseases. It was also successfully used to treat several infections. Mansour et al.(2016) evaluated BV as a treatment of Foot-and-Mouth disease in goats and Guinea pigs. There were improvements in hematological, biochemical and histopathological tests. The clinical signs decreased during the treatment period and the viral load significantly decreased. The antiviral effect of BV was also reported on Human Immunodeficiency Deficiency virus (HIV) (De-Clerq, 2000). BV has also been reported to have strong antibacterial activity against both Gram-negative and Gram positive bacteria and has also shown notable antifungal activity against Trichophyton men- tagrophytes and Trichophyton rubrum (Yu et al, 2012).

The present study aimed to investigate the scolicidal effect of bee venom on Echinococcus granulosus protoscolices.

\section{Materials and Methods}

E. granulosus hydatid cysts were collected from the lungs of naturally infected camels slaughtered at Cairo Governmental Abattoir. The solution inside each cyst was aspirated by a sterile syringe and allowed to settle in a sterile Falcon tube, where the protoscolices were precipitated and the supernatant was discarded. Protoscolices were then washed by sterile saline.

The viability of protoscolices was determined prior to experimental study by observation of motility of protoscolices and eosin exclusion test. A $0.1 \%$ eosin solution was added to the protoscolices solution in a ratio of 1:1. After 15 minutes, the viability of protoscolices was determined by observing the change of color under a light microscope. The numbers of viable/non-viable protoscolices were determined in 10 randomly chosen fields. Dead protoscolices absorb eosin and color red while viable protoscolices remain colourless. Viable protoscolices are mostly motile and show flame cell activity when put onto slides, covered with coverslips and counted under a light microscope. The protoscolices suspension was considered suitable for use when $>95 \%$ of protoscolices were viable.

Bee venom (BV): Bee venom was obtained from VACSERA (Egypt) under the trade name Abevac $(1 \mathrm{mg} / \mathrm{ml}$ vial) which is purified bee venom from Apis mellifera.

Effectiveness of BV on protoscolices: A drop of protoscolex-rich sediment containing 1500 protoscolices was added to each tube. Protoscolices were incubated with two concentrations of $\mathrm{BV}$ (50ppm \& 100ppm) for 30 minutes at $37^{\circ} \mathrm{C}$. At the end of the incubation period, the upper portion of the solution was discarded without disturbing the settled protoscolices and the protoscolices were washed with sterile saline. At least 
1500 protoscolices without exposure to $\mathrm{BV}$ were used as a control group. The scolicidal effect of BV was assessed by observation of motility, eosin exclusion test, cellular examination by light microscopy and ultrastructure examination by TEM. Apoptosis was determined by caspase- 3 immunohistochemistry.

Light microscopy: Both control and BVincubated protoscolices were fixed in $10 \%$ formalin, processed to paraffin blocks, cut, rehydrated and stained with hematoxylin and eosin $(\mathrm{H} \& \mathrm{E})$ and examined under the light microscope.

Transmission electron microscopy: TEM was used to study the ultrastructural changes in both control and BV-incubated protoscolices. Parasite material was fixed with $2.5 \%$ (v/v) glutaraldehyde in 0.1 cacodylate buffer, pH 7.2 for $1 \mathrm{hr}$. Fixed samples were washed twice in PBS and then post-fixed with $1 \%(\mathrm{v} / \mathrm{v})$ osmium tetroxide in $0.1 \mathrm{Mca}-$ codylate buffer for 30 minutes, $\mathrm{pH} \mathrm{7.2,} \mathrm{at}$ room temperature. They were dehydrated in increasing concentrations of ethanol. A final dehydration step was done in $100 \%$ propylene oxide and then samples were embedded in Epon 812 epoxy resin. Ultra-thin sections were cut by an ultra-microtome, mounted on upper grids and stained by uranyl acetate and lead citrate. Sections were examined under the TEM JEOL JEM-1200 EX II.

Immunohistochemistry: Caspase-3 anti-bo dies were diluted 1:300 and sections were subjected to high-pressure, high temperature antigen retrieval and subjected to staining procedures according to the kit instructions (Dako). The slides went through colour development with $\mathrm{DAB}$, counterstained with hematoxylin and observed under light microscopy. Yellow-brown staining in cytoplasm (may include nuclei) was considered as caspase-3 positive (Hu et al, 2011).

Statistical analysis: All experiments were performed at least 3 times. Data were presented as $\mathrm{M} \pm \mathrm{SD}$. Student's $t$-test was used to detect the statistical significance and was significant when $p$ value was $<0.05$ and highly significant when $p$ value was $<0.001$.

\section{Results}

Viability of protoscolices: Following isolation of protoscolices from hydatid cysts, eosin exclusion test revealed that $97 \%$ of protoscolices were viable and $80 \%$ showed distinct movements with flame cell activity. The majority of protoscolices were invaginated but around $20 \%$ were evaginated with clear rostellum and sucker. When eosin exclusion test was repeated after 30 minutes, $93 \%$ of control protoscolices were viable while only $54 \%$ of protoscolices incubated with 50ppm BV were viable with a highly significant statistical difference $(p<0.001)$. There was a $100 \%$ death rate in protoscolices incubated with $100 \mathrm{ppm}$ BV with a highly significant statistical difference $(p<0.001)$ (Figs. 1 \&2).

Morphological changes by light microscopy: Control protoscolices were clear and intact and the calcareous corpuscles were clear and bright with clear hooks and suction cups. Protoscolices that had been incubated with 50ppm BV showed vacuole formation, loss of suction cups and hooks, and discontinuation of the cuticle, while those incubated with 100ppm BV showed detachment and discontinuation of the cuticle and some were shrunken with complete loss of integrity and shape (Fig. 3).

Ultrastructural changes by TEM: Control protoscolices showed a tegument syncytium with aligned microtriches covered by a thick layer of periodic acid-schiff (PAS) positive substance. Internal parenchymal cells of protoscolices were round or oval with clear and large nuclei and centrally localized nucleoli. The chromatin was fine and smooth with a small amount of heterochromatin. Protoscolices incubated with BV showed apoptotic changes. These apoptotic changes were patchy in protoscolices incubated with $50 \mathrm{ppm}$ $\mathrm{BV}$ and generalized in those incubated with $100 \mathrm{ppm}$ BV. Protoscolices incubated with $50 \mathrm{ppm}$ BV showed loss of some of the PAS- 
positive material outside the tegument microtriches and condensed cytoplasm in the internal parenchymal cells with some apoptotic bodies and increased lipid droplets. Protoscolices incubated with 100ppm BV showed complete loss of the PAS-positive material as well as shedding of the microtriches. There was also increased vacuolation of the distant cytoplasm and increase in number of lipid droplets and apoptotic cells (Fig. 4).

Immunohistochemistry: Control protoscolices did not show any expression of caspase3 by immunohistochemistry. The expression of caspase- 3 in the protoscolices incubated with BV was significantly high. Protoscolices incubated with 50ppm BV showed large areas of brownish staining and those incubated in 100ppm BV showed complete brownish staining (Fig. 5). When PBS was used to replace the primary antibodies in the staining procedure, all the slides did not show any brownish staining which excludes false-positive results.

\section{Discussion}

Protoscolices are important targets of therapeutic agents to stop the development of hydatid cysts since they are capable of developing into adult worms in a definitive host or into new hydatid cysts in an intermediate host (Zou et al, 2009). Many scolicidal agents were used to inactivate hydatid cyst contents. However, the majority of these scolicidal agents have many side effects. The ideal scolicidal agent should have rapid and complete scolicidal effects with minimal local and systemic side effects (Altindis et al, 2004).

In the present study, BV was successfully used as an antibacterial, antiviral and antifungal agent (Leandro et al, 2015). It has also proven effective against protozoan infections. Crude BV was found to be lethalto Trypanosoma cruzi. Melittin that comprised $40-50 \%$ of the dry weight of BV, affected all Trypanosoma cruzi developmental forms, including the intracellular amastigotes. The ultrastructural changes induced by melittin suggested the occurrence of different programmed cell death or apoptosis pathways (Adade et al, 2012). Also, trypanocidal melittin concentrations were not toxic to host cells (Adade et al, 2013). Honeybee venom PLA2 disrupts cell membrane integrity by hydrolyzing 2-acyl bonds of membrane phospholipids, releasing lysophospholipids and fatty acids that themselves caused secondary membrane damage (Habermann, 1972). This enzyme also displayed bactericidal activity against Gram-negative enterobacteria, and was capable of lysing Trypanosoma brucei brucei in-vitro, at a concentration of $1 \mathrm{mg} / \mathrm{ml}$ (Boutrin et al, 2008). The efficacy of BV was also reported on Schistosoma mansoni infected mice. The results recorded reduction in the total worm burden, numbers of immature eggs and ova count in hepatic tissue in BV treated groups as compared to the infected group (Mohamed et al, 2016).

In the present study, the viability assay showed that only $54 \%$ of protoscolices were still viable after incubation with 50ppm BV for 30 minutes, while there was $100 \%$ death rate in the protoscolices incubated with $100 \mathrm{ppm} \mathrm{BV}$ for 30 minutes. The results of the viability assay coincided with the tissue damage observed at the structural level.

In the present study, BV caused distinct alterations in morphology of protoscolices by both light microscopy and TEM. Light microscopy showed many morphological changes in the protoscolices incubated with BV as compared to control ones. These changes included vacuole formation, loss of suction cups and hooks, detachment and discontinuation of the cuticle as well as shrinkage and complete loss of integrity and shape. The ultrastructural changes by TEM showed condensed cytoplasm in the internal parenchymal cells, increased vacuolation of the distant cytoplasm, increased in number of lipid droplets and presence of apoptotic cells in addition to loss of the microtriches. Similarly, when Trichomonas vaginalis trophozoites were incubated with BV for 30 minutes, it effectively inhibited their growth in a 
concentration-dependent manner. TEM of trophozoites showed cell membrane destruction and increased autophagic vacuoles (Kim et al, 2014). The morphological and ultra structural changes of protoscolices in this study were similar to changes reported by other drugs (Hu et al, 2011; Pensel et al, 2017).

In the present study, BV not only affected the cellular structure of protoscolices but also triggered apoptosis. Apoptosis was the most common form of eukaryotic cell death (Kerr et al, 1972). Caspase-3 is a cysteine aspartic acid protease that plays a central role in the execution-phase of apoptosis. It is actually the major terminal proteolytic enzyme in the process of apoptosis (Hu et al, 2011). The detection of caspase-3 was used to distinguish cell death pathways between apoptosis and necrosis. But, the exact mechanism of apoptosis in E. granulosus stages was not quite clear (Van-Cruchten and VanBroeck, 2002). In fact, understanding the exact mechanisms of apoptosis in E. granulosus stages will help the development of new treatment in the future. In the present study, the expression of caspase- 3 in protoscolices incubated with BV was significantly high while control protoscolices did not show any expression of caspase-3 which confirmed the occurrence of apoptosis. Similarly, melittin exerted its antifungal effect on Candida albicans via apoptosis (Park and Lee, 2010). Besides, melittin was proven to have an inhibitory effect on the proliferation of human cervical cancer cell lines through induction of apoptosis (Zarrinnahad et al, 2017). The induction of apoptosis by different scolicidal agents has been previously reported (Hu et al, 2011; Fabri et al, 2016; Xing et al, 2016).

\section{Conclusion}

The present study, proved the scolicidal effect of $\mathrm{BV}$ on E. granulosus protoscolices, but the exact mechanisms of anti-parasitic effect of BV need to be investigated. BV was shown to be a very promising scolicidal agent and further studies on its effects and safety in experimental animals are on-going and will be published in due time.

\section{References}

Adade, CM, Chagas, GS, Souto-Padron, T, 2012: Apis mellifera venom induces different cell death pathways in Trypanosoma cruzi. Parasitology 139, 11:1444-61.

Adade, CM, Oliveira, IR, Pais, JA, Souto-Padrón, T, 2013: Melittin peptide kills Trypanosoma cruzi parasites by inducing different cell death pathways. Toxicon 69:227-39.

Adas, G,Arikan, S, Kemik, O, Oner, A, Sahip, $\mathbf{N}$, et al,2009: Use of albendazole sulfoxide, albendazole sulfone, and combined solutions as scolicidal agents on hydatid cysts (in-vitro study). Wld. J. Gastroenterol. (WJG) 15, 1:112.

Altindis, M, Arikan, Y, Cetinkaya, Z, Polat, C, Yılmaz, S, et al, 2004: Octenidine hydrochloride in hydatid disease. J. Invest. Surg. 17, 1: 41-4.

Besim, HKKHOGÇ, Karayalcin, K, Hamamci, O, Güngör, C,Korkmaz, A, 1998: Scolicidal agents in hydatid cyst surgery. HPB Surg. 10, 6:347-51.

Boutrin, MC, Foster, HA, Pentreath, VW, 2008: The effects of bee (Apismellifera) venom phospholipase A2 on Trypanosoma brucei bruc$e i$ and enterobacteria. Exp .Parasitol.119, 2:24651.

Brunetti, E, Kern, P, Vuitton, DA, 2010: WHO/IWGE Expert consensus for the diagnosis and treatment of cystic and alveolar echinococcosis in humans. Acta Trop. 114, 1:1-16.

De Clercq, E, 2000: Current lead natural products for the chemotherapy of human immunodeficiency virus (HIV) infection. Med. Res. Rev. 20, 5:323-49.

El-Shazly, AM, Awad, SE, Hegazy, MA, Mohammad, KA, Morsy, TA, 2007: Echinococcosis granulosus/hydatidosis an endemic zoonotic disease in Egypt. J. Egypt. Soc. Parasitol. 37, 2: 609-22.

Fabbri, J, Maggiore, MA, Pensel, PE, Denegri, GM, Gende, LB, et al, 2016: In vitro and in vivo efficacy of carvacrol against Echinococcus granulosus. ActaTrop. 164:272-9.

Habermann, E, 1972: Bee and wasp venoms. Science 177, 4046:314-22.

Hosseini, SV, Ghanbarzadeh, K, Barzin, Z, Sadjjadi, SM, Tanideh, N, et al, 2006: In vitro protoscolicidal effects of hypertonic glucose on 
protoscolices of hydatid cyst. Kore. J. Parasitol. 44, 3:23-9.

Hu, H, Kang, J, Chen, R,Mamuti, W, Wu, G, et al, 2011: Drug-induced apoptosis of Echinococcus granulosus protoscoleces. Parasitol.Res. 109, 2:453-9.

Kayaalp, C, Balkan, M, Aydin, C, Ozgurtas, T, Tanyuksel, M, et al, 2001: Hypertonic saline in hydatid disease. Wld. J. Surg. 25, 8:975-9.

Kerr, JF, Wyllie, AH, Currie, AR, 1972: Apoptosis: A basic biological phenomenon with wideranging implications in tissue kinetics. Brit. J. Cancer 26, 4:239.

Kim, JH, Ryu, JS, Lee, MY, 2014: Inhibitory effect of bee venom on the growth of Trichomonas vaginalis. Toxicol. Environ. Hlth. Sci. 6, 1: 48-53.

Leandro, LF, Mendes, CA, Casemiro, LA, Vinholis, AH, Cunha, WR, et al, 2015: Antimicrobial activity of apitoxin, melittin and phospholipase A2 of honey bee (Apis mellifera) venom against oral pathogens. Ana. Acad. Brasil. Ciên. 87, 1:147-55.

Lee, YJ, Kang, SJ, Kim, BM, Kim, YJ, Woo, HD, et al, 2007: Cytotoxicity of honeybee (Apis mellifera) venom in normal human lymphocytes and HL-60 cells. Chemico-Biol. Interact. 169, 3: 189-97.

Mahmoudvand, H, Harandi, MF, Shakibaie, M, Aflatoonian, MR, Zia-Ali, N, et al, 2014: Scolicidal effects of biogenic selenium nanoparticles against protoscolices of hydatid cysts. Int. J. Surg.12, 5:399-403.

Mansour, AM, Elfiky, AA, Fahmy, A, Diab, A, 2016: Therapeutic effect of bee venom formulation in the treatment of FMD viral infection: Preclinical and clinical evaluation. Int. J. Sci. Res. Publica. 6, 6:711-29.

Moazeni, M, Alipour-Chaharmahali, MR, 2011: Echinococcus granulosus: in vitro effectiveness of warm water on protoscolices. Exp. Parasitol. 127, 1:14-7.

Moazeni, M, Nazer, A, 2010: In vitro effectiveness of garlic (Allium sativum) extract on scolices of hydatid cyst. Wld.J. Surg. 34, 11:267781.

Moazeni, M, Roozitalab, A, 2012: High scolicidal effect of Zataria multiflora on protoccoleces of hydatid cyst: an in vitro study. Compar. Clin. Pathol. 21, 1:99-104.

Mohamed, AH, El-Nabi, SEH, Bayomi, AE, Abdelaal, AA, 2016: Effect of bee venom or propolis on molecular and parasitological aspects of Schistosoma mansoni infected mice. J. Parasit. Dis. 40, 2:390-400.

Moon, DO, Park, SY, Choi, YH, Kim, ND, Lee, C, et al,2008: Melittin induces Bcl-2 and caspase-3-dependent apoptosis through downregulation of Akt phosphorylation in human leukemic U937 cells. Toxicon 51, 1:112-20.

Pak, SC, 2017: Chemical composition of bee venom. In: Bee Products: Chemical and Biological Properties, ed. J. Alvarez-Suarez (Springer).

Park, C, Lee, DG, 2010: Melittin induces apoptotic features in Candida albicans. Biochem. Biophys. Res. Commun. 394, 1:170-2.

Pensel, PE, Elissondo, N, Gambino, G, Gamboa, GU, Benoit, JP, et al, 2017: Experimental cystic echinococcosis therapy: In vitro and in vivo combined 5-fluorouracil/albendazole treatment. Vet. Parasitol. 245:62-70.

Pérez-Serrano, J, Casado, N, Rodriguez-Caabeiro, F, 1994: The effects of albendazole and albendazole sulphoxide combination-therapy on Echinococcus granulosus in vitro. Int. J. Parasitol. 24, 2:219-24.

Rahimi, MT, Ahmadpour, E, Esboei, BR, Spotin, A, Koshki, MHK, et al, 2015: Scolicidal activity of biosynthesized silver nanoparticles against Echinococcus granulosus protoscolices. Int. J. Surg. 19:128-33.

Rajabi, MA, 2009: Fatal reactions and methaemoglobinaemia after silver nitrate irrigation of hydatid cyst. Surg. Pract. 13, 1:2-7.

Sadjjadi, SM, 2006: Present situation of echinococcosis in the Middle East and Arabic North Africa. Parasitol. Int. 55:S197-202.

Sarkari, B, Rezaei, Z, 2015: Immunodiagnosis of human hydatid disease: where do we stand? Wld. J. Metho. 5, 4:185.

Sayek, I, Yalin, R, Sanaç, Y, 1980: Surgical treatment of hydatid disease of the liver. Arch. Surg. 115, 7:847-50.

Sharafi, SM, Sefiddashti, RR, Sanei, B, Yousefi, M, Darani, HY, 2017: Scolicidal agents for protoscolicesof Echinococcus granulosus hydatid cyst: Review of literature. J. Res. Med. Sci.: Offic. J. Isfahan Univ. Med. Sci. 22:92-9.

Tozar, E, Topcu, O, Karayalcin, K, Akbay, SI, Hengirmen, S, 2005: The Effects of cetrimide-chlorhexidine combination on the hepatopancreaticobiliary system. Wld. J. Surg. 29, 6:7548.

Van Cruchten, S, Van Den Broeck, W, 2002: Morphological and biochemical aspects of apoptosis, oncosis and necrosis. Anatom.Histol.Em- 
bryol.31, 4:214-23.

Xing, G, Wang, B, Lei, Y, Liu, C, Wang, Z, et al, 2016: In vitro effect of sodium arsenite on Echinococcus granulosus protoscoleces. Mol.

Biochem. Parasitol. 207, 2:49-55.

Yu, AR, Kim, JJ, Park, GS, Oh, SM, Han, C

S,et al,2012: Biochemistry: The antifungal activity of bee venom against dermatophytes. J. Appl. Biol. Chem. 55, 1:7-11.

Zarrinnahad, H, Mahmoodzadeh, A, Hamidi, MP, Mahdavi, M, Moradi, A, et al,2017:Apoptoticeffect of melittin purified from iranian honey bee venom on human cervical cancer HeLacell line. Int. J. Pept. Res. Therap. 69:1-8.

Zhou, J, Zhao, J, Zhang, S, Shen, J, Qi, Y, et al,2010: Quantification of melittin and apamin in bee venom lyophilized powder from Apis mellifera by liquid chromatography-diode array detector-tandem mass spectrometry. Anal. Biochem. 404, 2:171-8.

Zou, X, Wang, J, Zhao, H, Zhang, J, Wu, W, et al, 2009: Echinococcus granulosus: protoscolicidal effect of high intensity focused ultrasound. Exp.Parasitol.12, 4:312-6.

\section{Explanation of figures}

Fig. 1: Protoscolices after staining with $0.1 \%$ eosin. (a) Live protoscolices (Control) (b) Dead protoscolices after incubation with bee venom (BV) (x100).

Fig. 2: Percentage of live protoscolices after exposure to BV for 30 minutes.

Fig. 3: Morphological alterations of protoscolices stained with H\&E. (a-c) showed normal intact protoscolices with hooks and suction cups. (d-f) showed protoscolices incubated with 50ppm BV; (d) showed cuticle discontinuation (di). (e) showed suction cups loss (arrow). (f) showed vacuoles and cuticle discontinuation (di). (g-i) showed protoscolices incubated with 100ppm BV; (g) showed detachment of cuticle (de) and cuticle discontinuation (di). (h) showed shrunken protoscolex with deformity. (i) showed complete loss of integrity and shape (x400).

Fig. 4: Protoscolices by TEM. (a-b) Normal protoscolices showed syncytial layer of tegument (SL), PAS-positive material (arrow) and parenchymal cells (C) (a) (x2000) and (b) (x6000). (c-d) Protoscolices incubated with 50ppm BV (c) showed start of loss of PAS-positive material (arrow) (x2000). (d) showed start of loss of PAS-positive material (arrow), vacuolation of cytoplasm (V), condensed cytoplasm of parenchymal cells (C) and increased lipid droplets (l) (x4000). (e-h) Protoscolices incubated with 100ppm BV (e-f) showed shedding of microtriches (arrow) (e) (x2000) and (f) (x6000). (g) showed cytoplasm vacuolation (V) and condensed cytoplasm of parenchymal cells (C) (x8000). (h) showed apoptotic bodies (arrow) (x8000).

Fig. 5: Expression of caspase-3 in protoscolices by immunohistochemistry. (a) control protoscolices; (b) protoscolices incubated with 50ppm BV showed large areas of brownish staining; (c-d) protoscolices incubated with 100ppm BV showed complete brownish staining (x400).
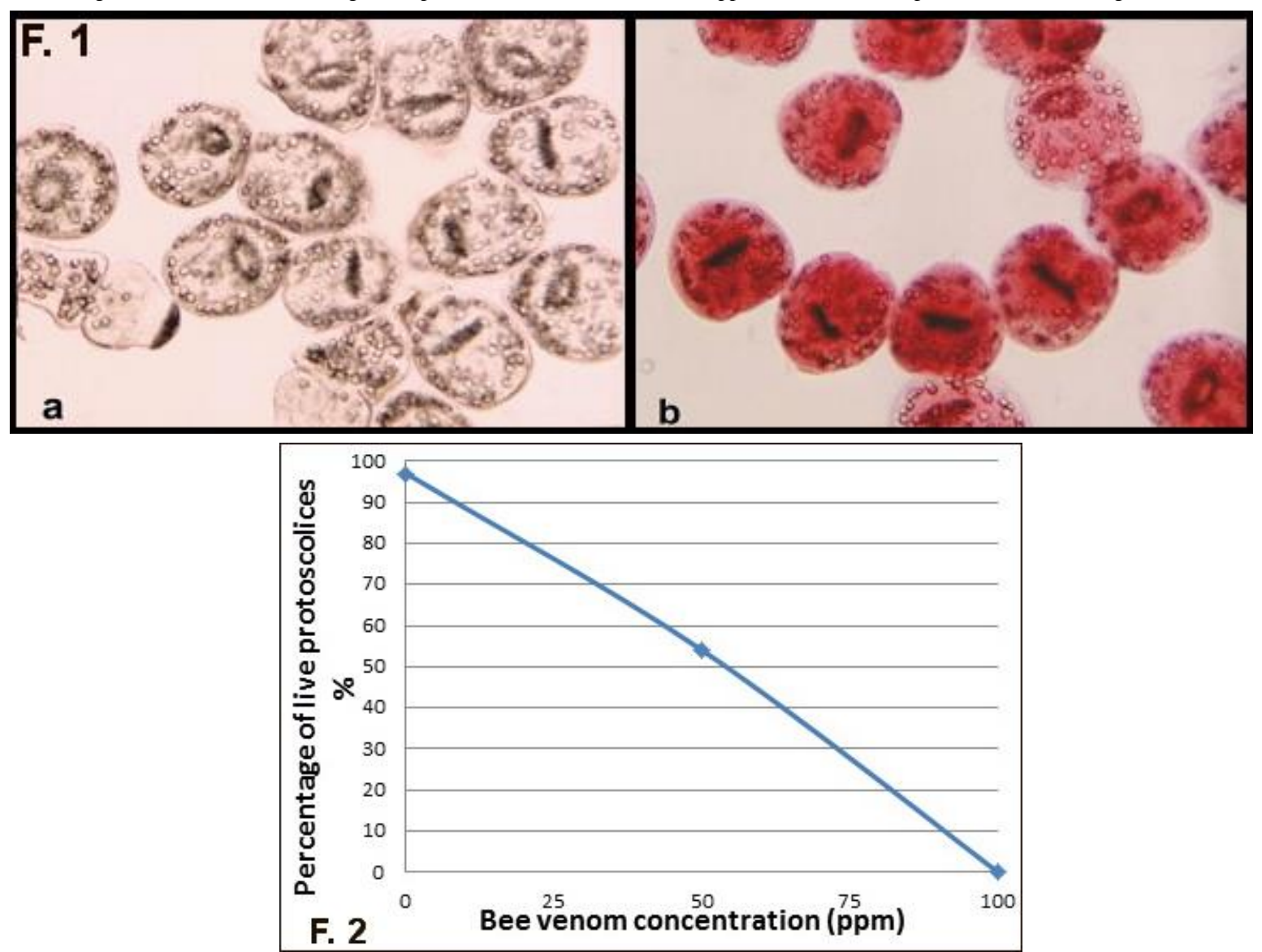


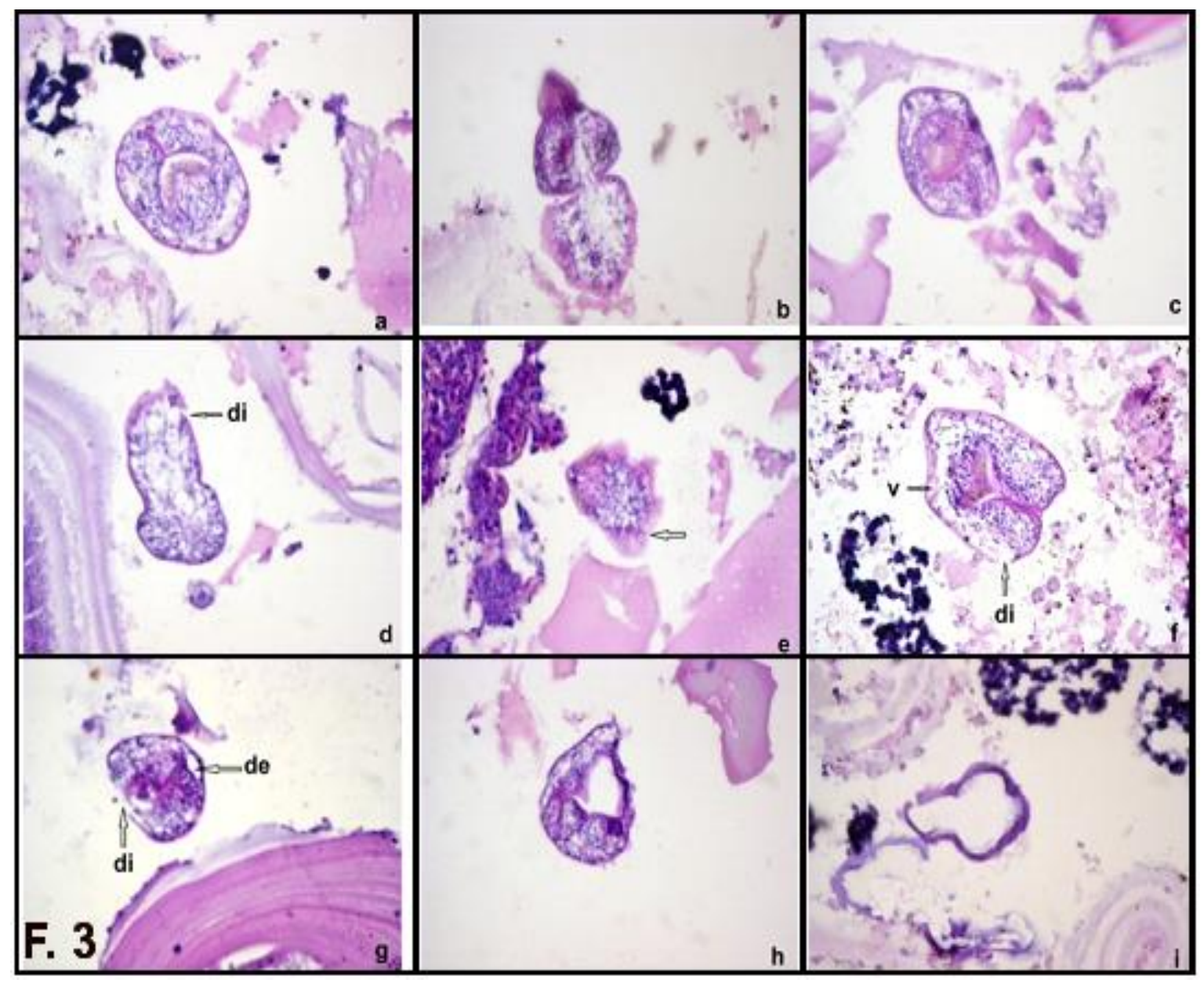



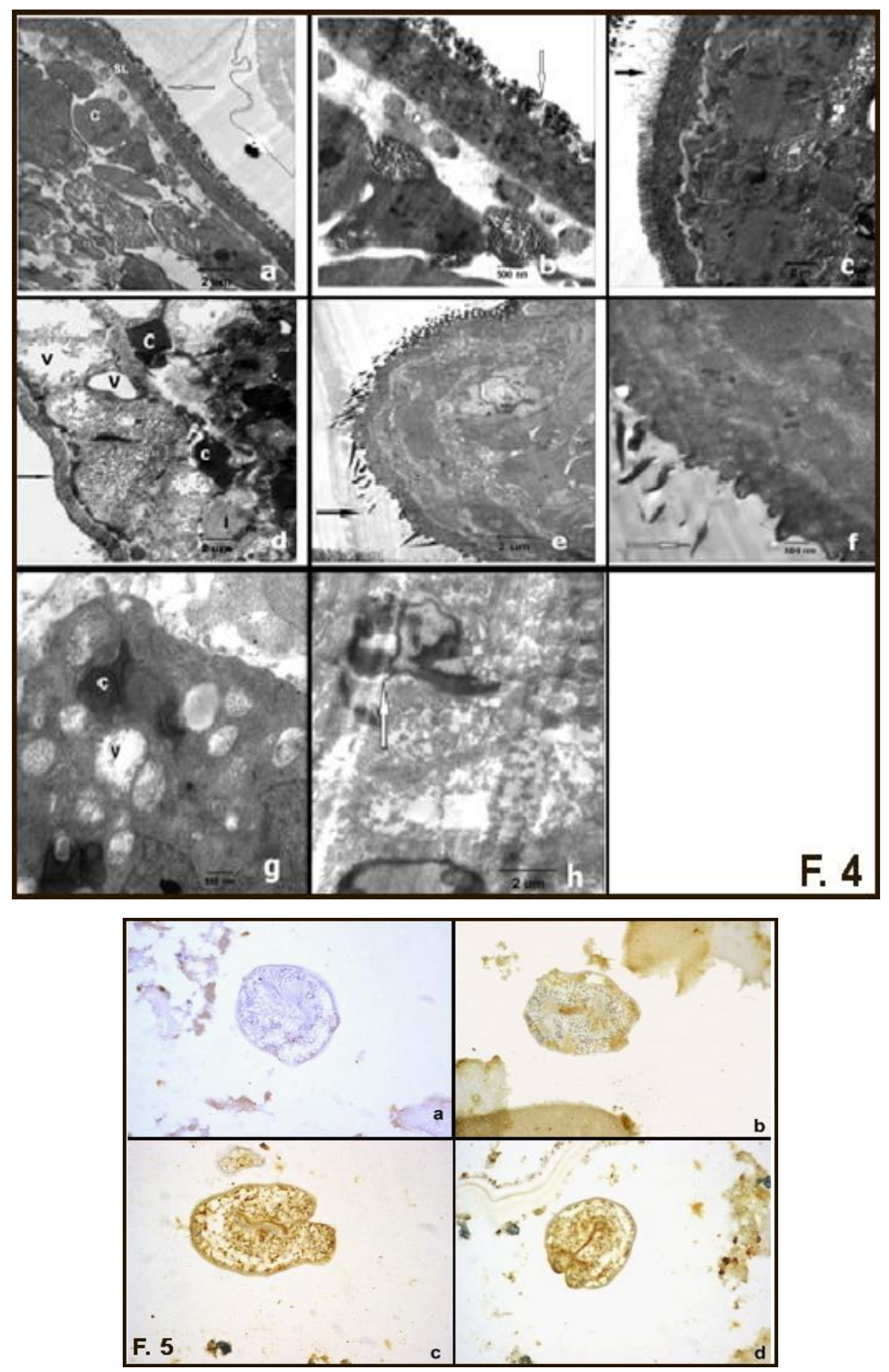